\title{
Magnetic fields and massive star formation
}

\author{
Richard M. Crutcher \\ Department of Astronomy, University of Illinois, Urbana, IL 61801, USA \\ email: crutcher@uiuc.edu
}

\begin{abstract}
Magnetic fields may be observed via the Zeeman effect, linear polarization of dust emission, and linear polarization of spectral-line emission. Useful parameters that can be inferred from observations are the mass-to-flux ratio $M / \Phi$ and the scaling of field strength with density. The former tells us whether magnetic fields exert sufficient pressure to provide support against gravitational contraction; the latter tells whether or not magnetic fields are sufficiently strong to determine the nature (spherical or disk geometry) of the contraction. Examples of massive star formation regions for which detailed observations have been made of magnetic field strengths and morphologies include DR21OH, OMC1, and S106; observational results for these regions and relevant results for the diffuse ISM and masers will be reviewed. Results are that the strength of interstellar magnetic fields remains invariant at $B \sim 6 \mu \mathrm{G}$ between $0.1 \mathrm{~cm}^{-3}<n(H)<10^{3} \mathrm{~cm}^{-3}$, but increases as $B \propto \rho^{0.4-0.5}$ for $10^{3} \mathrm{~cm}^{-3}<n\left(H_{2}\right)<10^{8} \mathrm{~cm}^{-3}$. Moreover, $M / \Phi$ is significantly subcritical (strong $B$ with respect to gravity) in diffuse $\mathrm{H}$ I clouds that are not self-gravitating, but becomes approximately critical in high-density molecular cloud cores. This suggests that GMCs form primarily by accumulation of matter along magnetic field lines, a process that will increase density but not magnetic field strength. How clumps in GMCs evolve will then depend critically on the $M / \Phi$ ratio in each clump.
\end{abstract}

Keywords. magnetic fields, polarization, stars: formation, ISM: clouds, ISM: magnetic fields

\section{Introduction}

The role that magnetic fields play in the formation of stars of all masses is far from clear, but this is especially true for high-mass stars. Magnetic fields may play significant or dominate roles in at least four areas: (1) in the formation of GMCs from which massive stars form, (2) in fragmentation to form stellar-mass clumps, (3) in the support of clouds against gravitational collapse, and (4) in transferring angular momentum outward from collapsing cores. Clarifying the role of magnetic fields can only come through observations of magnetic field strengths and morphologies in regions of massive star formation. Observations of magnetic fields in molecular clouds have now become a fairly routine procedure, and many new observational results have been obtained in the last few years. Most progress has been made in mapping polarized emission from dust, but many new Zeeman observations have also been made, together with the first detections of linearly polarized spectral lines. In this article I will review the observational results and focus on using the existent data to discuss the role of magnetic fields in massive star formation.

\section{Observational Techniques}

\subsection{Zeeman splitting}

The Zeeman effect is the only available technique for measuring directly magnetic field strengths in interstellar clouds (e.g. Crutcher, et al. 1993). To date Zeeman splitting in the interstellar medium has been detected only in spectral lines of $\mathrm{H} \mathrm{I}, \mathrm{OH}, \mathrm{CN}$, and $\mathrm{H}_{2} \mathrm{O}$, although searches continue to be made in other species. The reason for this 
limitation is that strong Zeeman splitting is only found in species with an unpaired electron, whose orbital angular momentum vector precessing in a magnetic field produces Zeeman splitting proportional to the Bohr magneton. Most interstellar molecules have paired electron spins, so any Zeeman splitting is proportional to the nuclear magneton, three orders of magnitude smaller. If the Zeeman components are completely separated, one gets the full strength of the magnetic field. Generally, however, the Stokes V spectrum reveals the sign (i.e., direction) and magnitude of the line-of-sight component $B_{\text {los }}$. For a large number of clouds whose magnetic fields are randomly oriented with respect to the observed line of sight, statistically $\bar{B}_{\text {los }}=\frac{1}{2}|\mathbf{B}|$.

\subsection{Polarization of dust emission}

Linear polarization of thermal emission from dust grains arises from elongated grains with their short axes aligned, generally with B (Lazarian 2004). The position angle of maximum emission will be perpendicular to $\mathbf{B}$ projected onto the plane of the sky, $B_{p o s}$, so maps of polarized dust emission give the morphology of $B_{\text {pos }}$. Chandrasekhar \& Fermi (1953) suggested that analysis of the small-scale randomness of magnetic field lines could yield estimates of the field strengths. Turbulent or MHD-wave motions will lead to irregular magnetic fields, and the stronger the regular field, the more it will resist being irregularized by turbulence. They showed that the magnitude of the irregularity of field lines could yield the regular field strength in the plane of the sky: $B_{\text {pos }}=Q \sqrt{4 \pi \rho} \delta V / \delta \phi$, where $\rho$ is the gas density, $\delta V$ is the velocity dispersion, $\delta \phi$ is the dispersion in polarization position angles, and $Q$ is a factor of order unity. Here we use $Q=0.5$, a calibration based on study of simulations of interstellar clouds (Ostriker, et al. 2001) that found that this method could yield reliable results in molecular clouds so long as $\delta \phi<25^{\circ}$.

\subsection{Spectral-line linear polarization}

Linear polarization may also arise in radio-frequency spectral lines formed in the interstellar medium, even when Zeeman splitting is negligible. Frequency-shifted $\sigma$ Zeeman components come from levels that only emit or absorb circularly polarized radiation parallel to the magnetic field or linearly polarized radiation perpendicular to the magnetic field, while the unshifted $\pi$ Zeeman component requires linearly polarized radiation parallel to the magnetic field. Therefore, anisotropic radiative excitation can unequally populate the magnetic sublevels and result in linearly polarized lines, parallel or perpendicular to the magnetic field. Which case holds depends on the angles between the line of sight, the magnetic field, and the direction of the anisotropic line optical depth. Also required is radiative dominating collisional excitation. Goldreich \& Kylafis (1981) and Kylafis (1983) discussed this effect in terms of a velocity gradient that would produce anisotropic trapping of molecular-line photons, which then would produce radiative excitation when re-absorbed. However, a continuum excitation source that occupies only a fraction of $4 \pi$ steradians may also produce anisotropic molecular excitation and linearly polarized line radiation. Spectral-line linear polarization may be used to probe magnetic field morphologies in molecular clouds, and the Chandrasekhar-Fermi method may be applied to maps of spectral-line linear polarization to estimate field strengths.

\subsection{Useful parameters}

There are two very useful parameters which can be inferred from observations of magnetic field strengths, the mass to magnetic flux ratio, $M / \Phi$, and the exponent $\kappa$ in a power-law representation of the scaling of field strength with density, $B \propto \rho^{\kappa}$.

$M / \Phi$ is a measure of the relative importance of gravity, acting to collapse a cloud, and magnetic pressure, acting to support it. $M / \Phi$ may be expressed in terms of the 
critical value at which gravity would be just balanced by magnetic support; we define $\lambda \equiv[M / \Phi]_{\text {observed }} /[M / \Phi]_{\text {critical }}$. Then $\lambda>1$ implies that the mass to flux ratio is supercritical, and the field cannot support the cloud against collapse; $\lambda<1$ implies that the mass to flux ratio is subcritical, and the field can support the cloud. Because $M / \Phi \propto N / B$, measuring column density $N$ and magnetic field strength $B$ allows inferring $M / \Phi$. However, since all three components of the vector $\mathbf{B}$ are seldom measured, and since $N$ is not generally measured along flux tubes, a statistical geometrical correction is necessary. For a disk geometry, appropriate for a cloud supported by a magnetic field, the corrected $\lambda$ is $\lambda_{C}=\lambda_{o b s} / 3$.

For $\mathbf{B}$ frozen into the gas, compression of the ISM purely perpendicular to $\mathbf{B}$ will result in $\kappa=1$; compression parallel to $\mathbf{B}$ produces $\kappa=0$. If a cloud becomes self-gravitating but the magnetic field is very weak, an approximately spherical collapse is expected (since $\mathbf{B}$ is too weak to impose a preferred direction on the collapse), in which case $\kappa=2 / 3$ is predicted (Mestel 1966). If magnetic fields support clouds and contraction occurs due to ambipolar diffusion in cores, $\kappa \approx 0.4-0.5$ is predicted (Mouschovias \& Ciolek 1999). Hence, the scaling exponent $\kappa$ in the $B \propto \rho^{\kappa}$ relation is an important diagnostic of the importance of the role of magnetic fields in cloud evolution.

\section{Observational Results}

\subsection{Diffuse Interstellar Medium}

Heiles \& Crutcher (2005) reviewed observations of magnetic fields in the diffuse interstellar medium. Results are that from the very low density Warm Ionized Medium through dense H I clouds, up to the extended regions of dark clouds, the magnetic field strength is approximately invariant: for $0.1 \mathrm{~cm}^{-3}<n(H)<10^{3} \mathrm{~cm}^{-3}, B \sim 6 \mu \mathrm{G}$ (see also Troland \& Heiles 1986). Exceptions hold in special cases, such as shells that have been compressed by supernova. Moreover, diffuse clouds with $\mathrm{n}(\mathrm{H} \mathrm{I}) \sim 50 \mathrm{~cm}^{-3}$ are significantly subcritical $\left(\lambda_{o b s}<1\right)$ but not self-gravitating.

\section{2. $D R 21 O H$}

Figure 1 shows results for the high-mass star formation region DR21OH; all three of the observational techniques discussed above have been employed. In millimeter-wave dust emission the main component of DR21OH consists of two compact cores with a total mass of $\sim 100 \mathrm{M}_{\odot}$ (Woody, et al. 1989). The two CN velocity components are each centered on a different one of the two compact cores. The region has signposts of highmass star formation: masers of $\mathrm{OH}$ (Norris, et al. 1982) and $\mathrm{H}_{2} \mathrm{O}$ (Genzel \& Downes 1977), and high-velocity outflows powered by the two compact cores (Lai, et al. 2003), but as yet no well-developed H II regions (since no continuum emission was detected at $15 \mathrm{GHz}$ (Johnston, et al. 1984)). The dust and CO 2-1 linear polarization toward the cores (Lai, et al. 2003) show that where both are detected at the same location, they are orthogonal. Since the polarization of dust emission is perpendicular to the magnetic field, this suggests that the magnetic field direction in $\mathrm{DR} 21 \mathrm{OH}$ is parallel to the CO polarization and therefore parallel to the major axis of $\mathrm{DR} 21 \mathrm{OH}$. The dust polarization data north of the cores suggest that $\mathbf{B}$ is along the minor axis to the north of the cores. This morphology could be explained by a large-scale poloidal field directed approximately north-south that has been twisted into a helical morphology toward the two high-density cores seen in figure 1. This twist could have been produced by the orbital motion of the double core; ultimately, as the twisting increased the local field strength, the field could transfer angular momentum outward. The $\mathrm{CO}$ and dust polarization maps suggest that magnetic fields are remarkably uniform throughout the region. Both the dust emission 

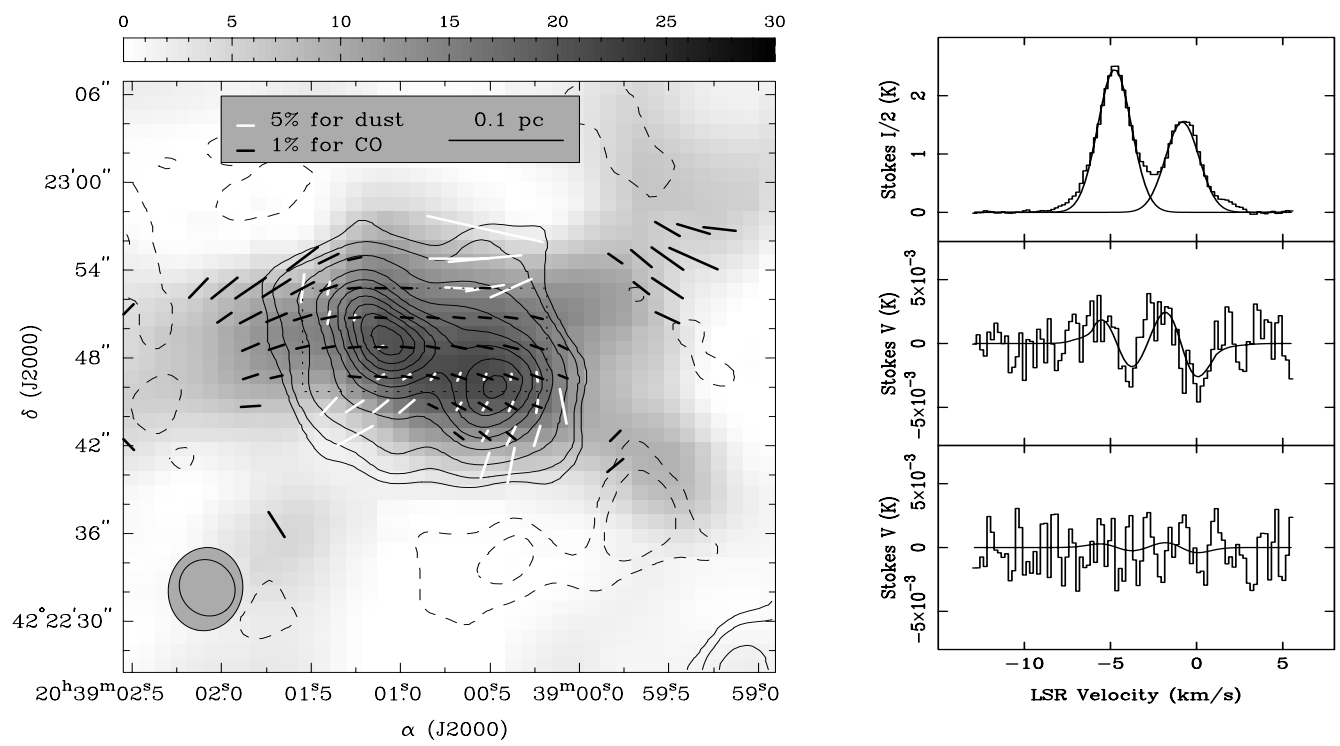

Figure 1. Left: BIMA map of DR21OH. Contours show the 1.3-mm dust emission, grey scale shows the CO 2-1 line emission integrated over velocity, white line segments show the dust polarization, and black line segments show the CO linear polarization. Right: IRAM 30-m CN 1-0 line profiles toward DR21OH. Observed data are histogram plots, fits are lines. Top panel shows the Stokes I spectrum with two Gaussians fitted. Middle panel shows the mean Stokes $\mathrm{V}$ spectrum for the four hyperfine components that have strong Zeeman splitting coefficients Z; the bottom panel shows the three components with weak $\mathrm{Z}$. $\mathrm{B}_{\text {los }}$ was fitted independently for the two Gaussian lines.

and the CN lines sample a density $n\left(H_{2}\right) \approx 1 \times 10^{6} \mathrm{~cm}^{3}$. The Chandrasekhar-Fermi technique yields $B_{\text {pos }} \approx 1 \mathrm{mG}$, compared with $B_{\text {los }}=-0.4 \pm 0.1 \mathrm{mG}$ and $B_{\text {los }}=-0.7 \pm 0.1$ $\mathrm{mG}$ inferred from the CN Zeeman detections (Crutcher, et al. 1999) shown in figure 1. Combining these results, $B_{\text {total }} \approx 1.2 \mathrm{mG}$ and $\mathbf{B}$ is at an angle $\theta \sim 55^{\circ}$ to the line of sight. If we accept that the total field strength is $1.2 \mathrm{mG}$ and make no geometrical correction, $\lambda \approx 2.0$, or supercritical. However, the column density along $\mathbf{B}$ would still be overestimated for disk geometry, by $1 / \cos 55^{\circ} \approx 1.7$; so $\lambda_{C} \approx 1.2$, or approximately critical. Uncertainties in $B_{\text {pos }}$ and $B_{\text {los }}$ are sufficiently large that $\theta$ is quite uncertain.

Cortes, et al. (2005) repeated the BIMA CO line mapping, but for the CO 1-0 line. A remarkable feature of this map is that the 1-0 line polarization is perpendicular to the 2-1 line polarization. The standard Goldreich-Kylafis theory cannot explain the two CO transitions having orthogonal polarizations. However, if there is a second source of anisotropy in the excitation of the magnetic substates of $\mathrm{CO}$, it is conceivable that the observations could be explained. Cortes, et al. (2005) investigated this possibility, and found that the flux of infrared photons produced by warm dust in the two massive cores could be sufficient to compete with $\mathrm{CO}$ line photon trapping excitation. If the $\mathrm{CO}$ polarization originates in an envelope surrounding the core, then the infrared photons would come from a limited area of sky - an anisotropic radiative excitation of CO. They carried out a detailed numerical study of excitation of the first 10 rotational states of $\mathrm{CO}$ by a combination of collisions, infrared photons from the central dust cores, and trapped CO line photons in the envelope. At low line optical depths, CO line trapping is not important and excitation of high J states of CO by infrared from the cores dominates; the prediction is that the line polarization is parallel to the magnetic field. At higher line optical depth, anisotropic photon trapping dominates, and line polarization 

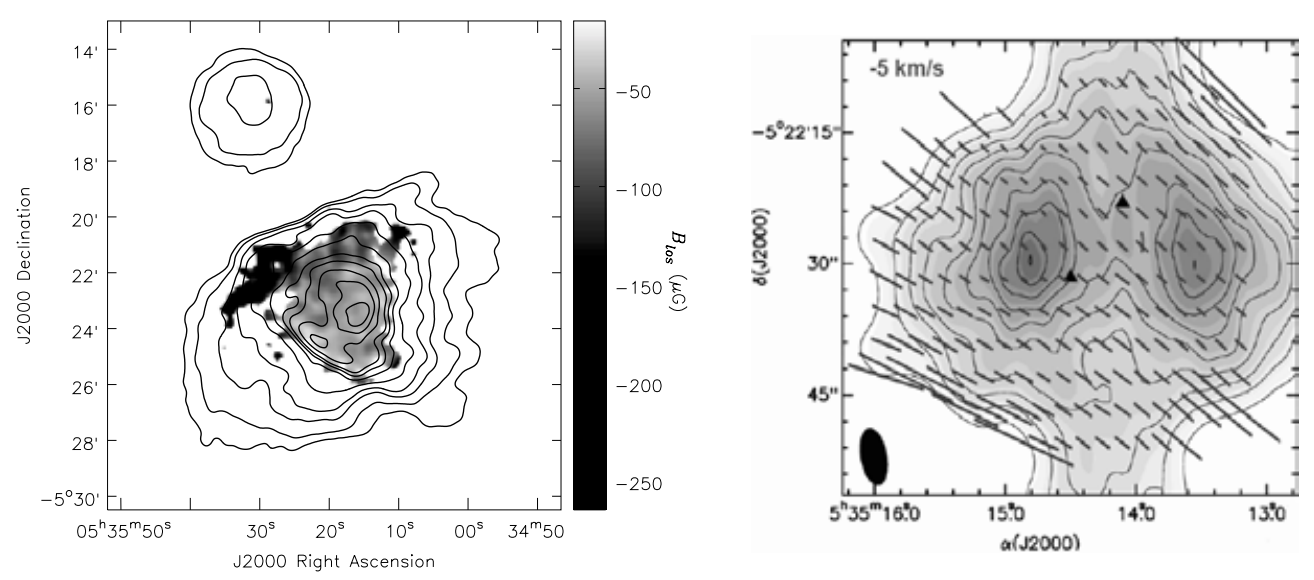

Figure 2. Left: VLA H I absorption-line map of $B_{l o s}$ (grey scale) in the "Orion Veil", in front of OMC1. Contours show the 21-cm continuum. Right: Combined BIMA and JCMT map of linearly polarized $\mathrm{J}=2-1 \mathrm{CO}$ emission in the blue-shifted line wing toward OMC1.

perpendicular to the magnetic field is predicted. Because the $\mathrm{J}=2$ and $\mathrm{J}=1$ states have different relative responses to the two sources of anisotropic excitation, it is possible for the domination to switch between continuum-photon excitation and line-photon trapping at different line optical depths in the two CO lines. The result of this modeling of the CO polarization has several astrophysical implications. First, the polarized CO must arise in low density gas, $\mathrm{n}\left(\mathrm{H}_{2}\right) \sim 100 \mathrm{~cm}^{-3}$, suggesting that an envelope region of the dense molecular cloud is being sampled. Since the CO and dust polarization maps show a similar morphology, the magnetic field morphology is very similar between the $10^{2} \mathrm{~cm}^{-3}$ envelope and the $10^{6} \mathrm{~cm}^{-3}$ core. Applying the Chandrasekhar-Fermi method to the $\mathrm{CO}$ polarization, $B \approx 10 \mu \mathrm{G}$ is found, consistent with a $\sqrt{\rho}$ scaling of $\mathrm{B}$ over these density extremes. Finally, the model helps to constrain the geometry of the region and its physical parameters.

\section{3. $O M C 1$}

Orion Molecular Cloud 1 (OMC1) is the closest region of OB star formation and probably the most thoroughly studied. Troland, et al. (2005) used the VLA to map the Zeeman effect in the absorption lines of $\mathrm{H} \mathrm{I}$ and $\mathrm{OH}$ (figure 2). Because the molecular cloud is behind the H II region, these results refer to less dense, mainly atomic gas (called the "veil"). Over most of the region, $B \sim-50 \mu \mathrm{G}$. However, toward the northeast, in a region called the "dark bay", where molecular absorption and emission is seen, $B \sim-200 \mu \mathrm{G}$. Because the "veil" has typically only $A_{V} \sim 1$, dust and molecular-line emission in this direction samples the much denser and massive background molecular cloud. Polarization of dust emission has been mapped at wavelengths from the far infrared to the millimeter. Dust emission polarization results (e.g., Houde, et al. 2004) show a fairly uniform polarization pattern with the inferred direction of $\mathbf{B}$ perpendicular to the NNE elongated axis of the molecular cloud, consistent with the magnetic field providing some support. Schleuning (1998) reported an "hourglass" morphology to B; since it is the tension of bent field lines that would provide support, an "hourglass" morphology is consistent with the molecular core being supported by the magnetic field. Rao, et al. (1998) produced higher resolution BIMA array maps of the dust polarization at $\lambda=2.6$ and $1.3 \mathrm{~mm}$. The magnetic field direction generally agreed with that of the lower resolution single-dish maps, except for a region to the southeast of $\mathrm{BN}$, where the position angles are shifted by almost $90^{\circ}$; 

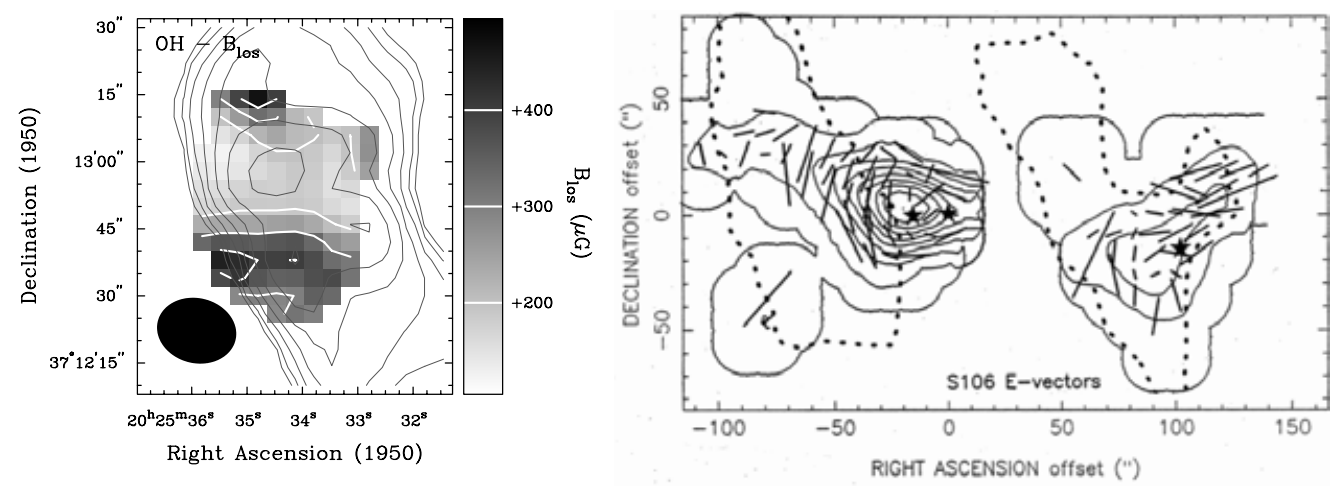

Figure 3. Left shows the VLA map of the S106 continuum (black contours) and line-of-sight magnetic field (white contours and grey scale. Right shows the SCUBA map of the dust polarization (line segments); the $(0,0)$ position is $\alpha_{1950}=202532.5, \delta_{1950}=371250$. The plane-of-sky magnetic field is at right angles to the dust polarization; dotted and solid contours are of CO and dust emission, respectively.

this may be due to mechanical grain alignment in the BN outflow. Rao, et al. (1998) also inferred $B_{\text {los }}<35 \mu \mathrm{G}$ in the $\mathrm{H}$ II region itself from the upper limit on Faraday rotation of the 1.3 and $2.6 \mathrm{~mm}$ position angles as the polarized emission from the background molecular cloud transits the ionized gas. Girart, et al. (2004) mapped linearly polarized CO J $=2-1$ line emission by combining BIMA and JCMT data in order to obtain full uv-plane coverage. Strong $(\sim 5 \%)$ polarization was generally seen in the line wings, with much weaker polarization in the line center. At blue-shifted velocities the polarization direction is very uniform (figure 2), while in the red-shifted line wing the polarization is generally similar, except for a region in the east and southeast, where the position angle is $\sim 90^{\circ}$. The position angles of the $\mathrm{CO}$ and dust polarization generally do not agree in detail, although both are generally in the range $20^{\circ}-50^{\circ}$. Since the $\mathrm{CO}$ polarization is sampling much lower density regions than is the dust polarization (see above discussion of $\mathrm{DR} 21 \mathrm{OH}$ ), this lack of detailed agreement is not surprising. However, the generally uniform polarization patterns suggest a rather uniform magnetic field morphology that is not entirely different in the two regions. Finally, Crutcher, et al. (1999) detected the Zeeman effect in the $\mathrm{N}=1-0$ lines of $\mathrm{CN}$ at $2.6-\mathrm{mm}$, inferring $B_{\text {los }} \approx 0.4 \mathrm{mG}$. The $\mathrm{CN}$ lines sample $n\left(H_{2}\right) \sim 10^{6} \mathrm{~cm}^{-3}$ and $N\left(H_{2}\right) \approx 2 \times 10^{23} \mathrm{~cm}^{-2}$; these data yield $\lambda_{C} \approx 1.0-$ or a critical mass-to-flux ratio.

\section{4. $S 106$}

Sharpless 106 is a compact, bipolar H II region embedded in an extended ( $\sim 3 \mathrm{pc})$ molecular cloud with $M \sim 10^{3} M_{\odot}$ and $n\left(H_{2}\right) \sim 10^{3} \mathrm{~cm}^{-3}$. The H II region is about $0.5 \mathrm{pc}$ in length with a cylindrical morphology bisected by a central dust lane. A small young star cluster is seen in the near infrared, with the most massive star (IRS 4) apparently being a pre-main sequence massive $\left(>15 M_{\odot}\right)$ O-type star that has formed by an accretion disk process (Noel, et al. 2005). Roberts, et al. (1995) mapped the free-free continuum and $\mathrm{OH}$ and $\mathrm{H} \mathrm{I}$ absorption lines with the VLA and measured the Zeeman effect in $\mathrm{OH}$ and $\mathrm{H}$ I (figure 3). The $\mathrm{OH}$ absorption lines show two strong components, with radial velocities of $\sim-1.5 \mathrm{~km} \mathrm{~s}^{-1}$ and $\sim-4 \mathrm{~km} \mathrm{~s}^{-1}$ and line widths of $\sim 2 \mathrm{~km} \mathrm{~s}^{-1}$ and $\sim 5 \mathrm{~km} \mathrm{~s}^{-1}$, respectively. The narrow line at $\sim-1.5 \mathrm{~km} \mathrm{~s}^{-1}$ corresponds in velocity and width with the $\mathrm{CO}$ emission seen over the extended molecular cloud, while the broad, blue-shifted line is seen only toward the H II region and appears to be molecular gas shocked by the H II 
region. The narrow line therefore traces molecular gas that has not yet been affected by the $\mathrm{H}$ II region. It is seen strongly in $\mathrm{OH}$ absorption only toward the eastern edge of the $\mathrm{H}$ II region near the dust lane. Molecular emission-line maps show a dense $\left(\sim 10^{5} \mathrm{~cm}^{-3}\right)$ clump that corresponds to the $\mathrm{OH}$ absorption. Roberts, et al. (1995) therefore argued that their $\mathrm{OH}$ Zeeman map of this narrow line sampled the magnetic field in an undisturbed dense clump in this high-mass star formation region. Their map of $B_{\text {los }}$ is shown in figure 3 . $B_{\text {los }}$ has maximum of almost $+0.5 \mathrm{mG}$ south of the dust lane that crosses the $\mathrm{H}$ II region, decreases to $\sim 0.1 \mathrm{mG}$ in the dust lane, and increases to $\sim 0.4 \mathrm{mG}$ north of the lane. For the dense clump $\lambda_{C} \approx 0.3$, which would be a subcritical mass-to-flux ratio. Vallée \& Fiege (2005) mapped dust emission polarization with SCUBA (figure 3). Their map shows that the plane-of-sky field is roughly parallel to the long axis of the H II region at the extreme east and west positions where dust polarization can be detected, but that the field is twisted by $\sim 90^{\circ}$ in the central region. A 3D field morphology that can explain all of the data is that the position angle of the plane-of-sky magnetic field direction is $\sim 20^{\circ}$ over the extended molecular cloud, with the line-of-sight component of the field pointing away from us; the value of the angle between the line-of-sight and $\mathbf{B}$ is unknown. Toward the dust lane (disk) projected on the H II region, the field has been twisted by $\sim 90^{\circ}$ into a toroidal configuration, perhaps by the rotation of the contracting core that formed IRS 4 and the associated stellar cluster. This is similar to the situation found toward DR21OH.

\subsection{Maser Zeeman Observations}

$\mathrm{OH}$ masers are found associated with the early stage of massive star formation, with maser spots coming from the dense $\left(\sim 10^{7} \mathrm{~cm}^{-3}\right)$ molecular envelope surrounding the massive star. Because of their brightness, they serve as signposts identifying sites of recently formed massive stars, and can be used to study kinematic and physical conditions in the dense molecular material. The ground state ${ }^{2} \Pi_{3 / 2}, J=3 / 2 \mathrm{OH}$ masers sometimes have clearly identifiable Zeeman pairs, that imply milligauss magnetic field strengths. Here $B_{t o t}$ is measured since the two Zeeman pairs are (generally) separated.

Argon, et al. (2000) surveyed 91 regions with the VLA A-array in both senses of circular polarization simultaneously, in order to identify Zeeman pairs. Fish, et al. (2003) analyzed this sample and found more than 100 Zeeman pairs in more than 50 regions. Field strengths range from $\sim 0.1 \mathrm{mG}$ to $\sim 10 \mathrm{mG}$. They derived a magnetic field direction for each massive star formation region and looked for correlations, such as the correlations between maser field directions and the large-scale Galactic field suggested by Davies (1974) based on a much smaller data set. The more complete data did not show this correlation, which if present would have required a preservation in field direction between the very diffuse and the very dense gas.

Excited state $\mathrm{OH}\left({ }^{2} \Pi_{3 / 2}, J=5 / 2\right.$ and $\left.J=7 / 2\right)$ maser lines were observed by Caswell (2003, 2004). The excited-state masers tend to have fewer components and "cleaner" Zeeman pairs than the ground-state masers. Field strengths are similar to those found in the ground-state maser lines.

Fiebig \& Güsten (1989) detected Zeeman splitting in the $\left(6_{16}-5_{23}\right) \mathrm{H}_{2} \mathrm{O}$ maser lines toward W 3, Orion KL, W49N, and S140 and inferred field strengths up to $50 \mathrm{mG}$. $\mathrm{H}_{2} \mathrm{O}$ masers probe densities $\sim 10^{8-9} \mathrm{~cm}^{-3}$. Because $\mathrm{H}_{2} \mathrm{O}$ does not have an unpaired electron, the Zeeman splitting is proportional to the nuclear magneton, and only $B_{\text {los }}$ could be measured. Sarma et al. (2002) used the VLA to continue these studies, finding $B_{l o s} \approx 13-$ $49 \mathrm{mG}$ in four massive star formation regions. They argued that the masers arise in C-shock regions, and that the magnetic and turbulent energies are close to equilibrium. Sarma et al. (2001) used the VLBA to map four $\mathrm{H}_{2} \mathrm{O}$ maser spots in W3 IRS5, finding 

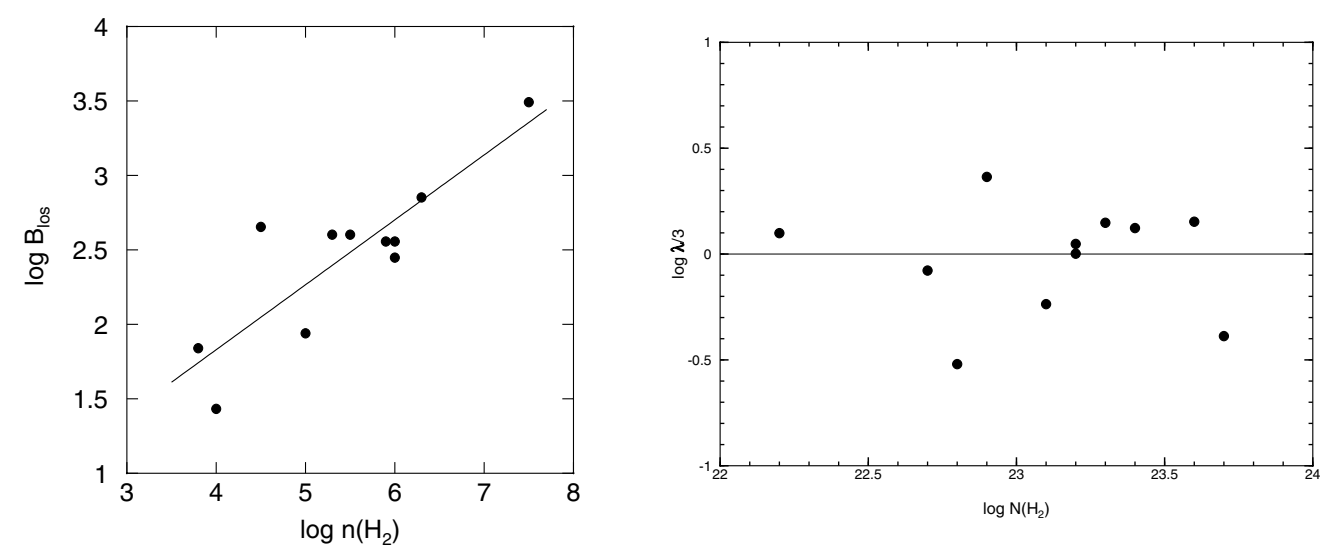

Figure 4. Left shows the high-mass star formation region magnetic field strengths plotted against the volume density; the linear fit has a slope $\kappa=0.44 \pm 0.09$. Right shows the observed mass to magnetic flux ratios, divided by 3 to correct for projection bias, in units of the critical values; $\log \lambda_{C}=0$ is critical.

that $B_{l o s}$ varied by a factor of three over 150 au but did not change sign. This might be expected if the masers and magnetic field are entrained in a coherent outflow.

Unfortunately, uncertainties in the maser column and volume densities limit their usefulness in determining $\lambda$ and $\kappa$.

\section{Discussion}

\subsection{Scaling}

At low densities $\left(n \sim 0.1-100 \mathrm{~cm}^{-3}\right)$ it has been clear for some time that there is no correlation of $|\mathbf{B}|$ with $\rho$ (Troland \& Heiles 1986); $B \approx 6 \mu \mathrm{G}$ is the mean value. At densities above $10^{3} \mathrm{~cm}^{-3}$, magnetic field strengths are higher. Figure 4 shows $B_{\text {los }}$ plotted against volume density for the 11 high-mass star formation clouds. One finds $\kappa=0.44 \pm 0.09$ for the $B \propto \rho^{\kappa}$ scaling relationship. Fields in still denser regions sampled by masers also seem to scale in this way, although the densities in the masing regions are not precisely known. This $\kappa$ agrees with the strong magnetic support, ambipolar diffusion model and not with the very weak field, spherical collapse prediction of $\kappa=2 / 3$. However, one must remember that these objects are remnant clumps in regions where high-mass star formation has already occurred. The progenitor clumps from which those stars formed may well have had more mass relative to the magnetic support and have collapsed at near a free-fall rate, with $B$ scaling closer to $\rho^{2 / 3}$ during that collapse phase. In any case, the magnetic field data do show that magnetic fields are sufficiently strong in regions of massive star formation to be dynamically important.

\subsection{Mass-to-flux ratio}

Figure 4 shows the geometry-corrected mass-to-flux ratios $\lambda_{C}$ from Zeeman detections for molecular clouds associated with high-mass star formation (Crutcher 1999). The observations are distributed roughly equally above and below the $\lambda_{C}=1$ line that divides subcritical and supercritical $M / \Phi$ ratios for disk geometries. Therefore, the data suggest that $\bar{\lambda} \approx 1$; that is, the typical mass to magnetic flux ratio is approximately critical. Although only data for high-mass star formation regions are shown in figure 4, molecular clouds associated with low-mass star formation show a similar result - the 


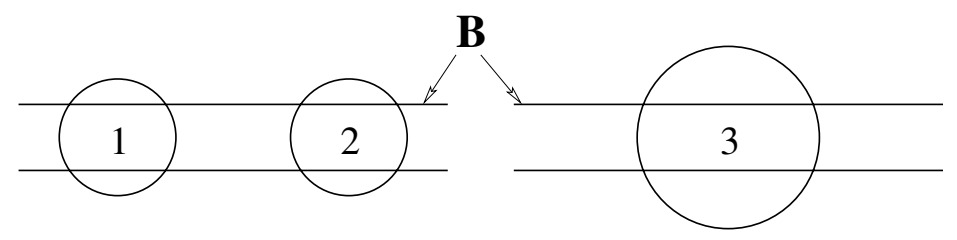

Figure 5. Cartoon of how formation of molecular clouds by accumulation along flux tubes can increase the observed mass-to-flux ratio.

mass-to-flux ratios are approximately critical. Again, however, one must remember that what we can observe are the remnant dense molecular clumps in high-mass star formation regions. Other clumps which were present in these regions in the past collapsed to form the high-mass stars that are now seen. The mass-to-flux ratios in these clumps must have been supercritical for them to have collapsed; we have no information about whether they were formed supercritical and collapsed on a fast time scale, or had a period of magnetic support with ambipolar diffusion overcoming magnetic support in the cores.

The fact that the mass-to-flux ratio is approximately critical in molecular clumps within GMCs has an important implication for GMC formation and evolution. The field in H I clouds, with $n(H I) \sim 50 \mathrm{~cm}^{-3}$, is significantly subcritical. Hence, the H I data suggest that the precursors to molecular clouds are significantly magnetically subcritical, and the formation process has resulted in critical mass-to-flux ratios in molecular clumps in GMCs. As noted above, compression perpendicular to field lines will result in $B$ scaling linearly with $\rho$, while compression parallel to field lines results in no change in $B$ as $\rho$ is increased. This suggests that GMCs are formed by material accumulating along flux tubes to form dense clouds (e.g., Hartmann, et al. 2001). Although this would not actually increase the mass-to-flux ratio in a flux tube, observers of individual $\mathrm{H} \mathrm{I}$ clouds in the flux tube would infer a lower $\lambda$ than would be found after $\mathrm{H}$ I clouds aggregate to form a single dense molecular cloud. Figure 5 shows how this change in $\lambda$ may have taken place. The H I clouds 1 and 2 could (for example) have observed $\lambda=1 / 2$ but be located in the same flux tube. Flows along the flux tube, either driven by turbulence or by the Parker instability (Parker 1966), could cause mergers of H I clouds, producing clouds such as cloud 3, that would have twice the mass but the same magnetic field strength. Hence, the observed $\lambda$ of cloud 3 would be 1 . A combination of accumulation of matter within flux tubes, turbulence driven ambipolar diffusion (Heitsch, et al. 2004), and gravity driven ambipolar diffusion (Mouschovias \& Ciolek 1999) may all be important at different stages in molecular cloud formation and collapse.

\section{Conclusions}

Data on magnetic fields in regions of high-mass star formation show that fields are sufficiently strong to be dynamically important. They appear to play a role in the formation of molecular clouds, for the fact that the density increases but the magnetic field strength does not strongly suggests that compressional motions are primarily along field lines. In the molecular clumps which remain in GMCs that have undergone formation of massive stars, the mass-to-flux ratio is approximately critical; such clumps can further contract in their cores and form stars through the action of ambipolar diffusion. The most massive stars, that have already formed, may have been the result of mass accumulation that produced supercritical clumps, that could evolve on a dynamical time scale to form massive stars. The scaling of field strength with density for individual clouds further supports the picture of dynamically important magnetic fields. But, as is often the case, 
the data on magnetic fields in molecular clouds remains quite sparse. A definitive answer to the question of what role due magnetic fields play in massive star formation awaits further observational and theoretical progress.

This work was partially supported by NSF grant AST 02-05810. I thank Tom Troland for an enjoyable and productive collaboration on the study of interstellar magnetic fields.

\section{References}

Argon, A. L., Reid, M. J., \& Menten, K. M. 2000, ApJS 129, 159

Caswell, J. L. 2003, MNRAS 341, 551

Caswell, J. L. 2004, MNRAS 352, 101

Chandrasekhar, S. \& Fermi, E. 1953, ApJ 118, 113

Cortes, P. C., Crutcher, R. M., \& Watson, W. D. 2005, ApJ, in press

Crutcher, R. M., Troland, T. H., Goodman, A. A., Heiles, C., Kazès, I., \& Myers, P. C. 1993, ApJ 407, 175

Crutcher, R. M., Troland, T., Lazareff, B., Paubert, G., \& Kazès, I. 1999, ApJ 514, L121

Crutcher, R. M. 1999, ApJ 520, 706

Davies, R. D. 1974, IAUS 60, 275

Fiebig, D. \& Güsten, R. 1989, AAp 214, 333

Fish, V. L., Reid, M. J., Argon, A. L., \& Menten, K. M. 2003, ApJ 596, 328

Genzel, R. \& Downes, D. 1977, AApS 30, 145

Girart, J. M., Greaves, J. S., Crutcher, R. M., \& Lai, S.-P. 2004, Ap \& SS 292, 119

Goldreich, P. \& Kylafis, N. D. 1981, ApJ 243, L75

Hartmann, L., Ballesteros-Paredes, J., \& Bergin, E. A. 2001, ApJ 562, 852

Heiles, C. \& Crutcher, R. 2005, in Cosmic Magnetic Fields, edited by R. Wielibinski \& R. Beck. Springer, in press

Heitsch, F., Zweibel, E. G., Slyz, A. D., \& Devriendt, J. E. G. 2004, ApJ 603, 165

Houde, M., Dowell, C. D., Hildebrand, R. H., Dotson, J. L., Vaillancourt, J. E., Phillips, T. G., Peng, R., \& Bastien, P. 2004, ApJ 604, 717

Johnston, K. J., Henkel, C., \& Wilson, T. L. 1984, ApJ 285, L85

Kylafis, N. D. 1983, ApJ 275, 135

Lai, S. P., Girart, J. M., \& Crutcher, R. M. 2003, ApJ 598, 392

Lazarian, A. 2003, JQSRT 79-80, 881

Mestel, L. 1966, MNRAS 133, 265

Mouschovias, T. Ch. \& Ciolek, G. E. 1999, in The Origin of Stars and Planetary Systems, edited by C. J. Lada \& N. D. Kylafis, Kluwer, Dordrecht, pp. 305-339

Noel, B., Joblin, C., Maillard, J. P., \& Paumard, T. 2005, AAp 436, 569

Norris, R. P., Booth, R. S., Diamond, P. J., \& Porter, N. D. 1982, MNRAS 201, 191

Parker, E. N. 1966, ApJ 145, 811

Ostriker, E. C., Stone, J. M., \& Gammie, C. F. 2001, ApJ 546, 980

Rao, R., Crutcher, R. M., Plambeck, R. L., \& Wright, M. C. H. 1998, ApJ 502, L75

Roberts D. A., Crutcher R. M., \& Troland T. H. 1995, ApJ 442, 208

Sarma, A. P., Troland, T. H., \& Romney, J. D. 2001, ApJ 554, L217

Sarma, A. P., Troland, T. H., Crutcher, R. M., \& Roberts, D. A. 2002, ApJ 580, 928

Schleuning, D. A. 1998, ApJ 493, 811

Troland, T. H. \& Heiles, C. 1986, ApJ 301, 339

Troland, T. H., Brogan, C., Crutcher, R. M., \& Goss, W. M. 2005, ApJ, to be submitted

Vallée J. P. \& Fiege, J. D. 2005, ApJ, in press

Woody, D. P., Scott, S. L., Scoville, N. Z., Mundy, L. G., Sargent, A. I., Padin, S., Tinney, C. G., \& Wilson, C. D. 1989, ApJ 337, L41 